\title{
Serum Lipidomic Biomarkers from Patients with Prostate Pathology Identified by High Performance Liquid Chromatography Coupled with Mass Spectrometry
}

\author{
Andrei LAZĂR ${ }^{1}$, Florina ROMANCIUC ${ }^{1}$, Nicolae CRIŞAN ${ }^{2}$, Carmen SOCACIU ${ }^{1,3}$ \\ ${ }^{1}$ University of Agricultural Sciences and Veterinary Medicine, 3-5 Mănăştur Street, Cluj-Napoca, \\ ${ }^{2}$ University of Medicine and Pharmacy "Iuliu Hatieganu" Cluj-Napoca \\ ${ }^{3}$ Center for Applied Biotechnology CCD-BIODIATECH Cluj-Napoca, Romania \\ * corresponding author: andrei.lazar@usamvcluj.ro
}

Bulletin UASVM Animal Science and Biotechnologies 73(2)/ 2016

Print ISSN 1843-5262; Electronic ISSN 1843-536X

DOI:10.15835/buasvmcn-asb: 12205

\begin{abstract}
Lipidomics can offer an instant picture of the lipophilic metabolites from tissues and biofluids and can indicate the presence of different pathologies, such as hyperplasia or different types of cancers. Related to these pathologies, Prostate Serum Antigen (PSA), proved to have a low grade prediction for an accurate diagnosis. Meanwhile, untargeted or targeted metabolomics became a useful technology to discover new biomarkers for a better diagnostic. The aim of this study is to realize an adequate procedure based on liquid chromatography coupled with mass spectrometry (HPLC-MS) to determine the profile of lipids from blood serum, followed by adequate biostatistics. Blood samples, obtained from healthy men and patients with prostate benign hyperplasia, post-biopsy cancer and post-surgery cancer were processed for extraction of lipids with Bligh \& Dyer method, and subjected to HPLC-ESI(+)QTOF-MS measurements. TofControl 3.2 and Data Analysis 4.2 software (Bruker Daltonics) were used for the control of the instrument and data processing. To process the matrix data, Profile Analysis 2.0 software was applied for alignment and advanced bucketing and then, the multivariate analysis (PCA and Cluster Analysis) using Unscrambler 10.1 software. The statistical unsupervised analysis based on PCA scores and loadings, showed a good discrimination between the two cancer groups of patients (after biopsy and after surgery) and for benign hyperplasia patients against controls, based on the comparison of peak areas. The molecules responsible for such discriminations were identified to be mainly represented by lysophospatidylcholines. By Cluster Analysis, the dendograms showed good statistical clustering of samples, especially for cancer patients against controls and less clustered for hyperplasia. Finally, one can consider that molecules belonging to phospholipid family and diacyl / triacylglycerides or ceramides or carnitines can be considered potential biomarkers for hyperplasia and prostate cancer.
\end{abstract}

Keywords: lipidomics, prostate hyperplasia, cancer, mass spectrometry, biomarker

\section{INTRODUCTION}

Prostate cancer (PCa) became a common malignancy and the second leading cause of cancer deaths in men (Ferlay et al., 2013). Recent studies of metabolite profiling in the prostate tissue, in blood and urine of patients with $\mathrm{PCa}$ showed the potential of metabolomics to improve diagnosis and provide answers regarding tumor invasiveness (Osl et al., 2008; Lochov et al. 2010; Teahan et al. 2011; Zhang et al. 2014), a better detection and prediction being crucial for the patient survival.

Metabolic biomarkers are small molecules (with molecular weight under 5000 Da) specific to tissues, cells or biofluids, wich can indicate the presence and evolution of a certain metabolic 
pathway or pathology, or to reflect the effects of applied treatments (Mishra and Verma, 2010).

Few prostate cancer biomarkers for early stage detection are available, the most known being the Prostate Serum Antigen (PSA) an androgen regulated protein biomarker. By its low predictability, PSA cannot have a good prognostic value, can identify less then $70 \%$ of $\mathrm{PCa}$, does not distinguish sufficiently between malignant and benign diseases (prostatitis and prostatic hypertrophy) (Vicini et al., 2005)

New reliable, predictive biomarkers are necessary for early and strength diagnosis beside the biopsy, as a gold standard (Verma and Banerjee 2015).

Lipidomics, a branch of metabolomics specifically focuses on lipids and their metabolites in physiologic or pathologic states (Han, 2009). These molecules are the structural component of the cell membrane embedded with various protein complexes with different functions in cell signaling as a substrate, product or co-factor in the biochemical reactions. Lipids are involved in a variety and complex physiological processes like signaling membrane, reserve energy and endocrine action (Feng and Prestwich, 2005; Wenk, 2005; Wolf and Quinn, 2008). Therefore, lipidomics can be used to improve the diagnostic and prognosis by small lipid metabolites which are expected to reflect the alterations in the cellular and tissular metabolism (Punglia 2003). Recent studies show the lipidomic profile changes in the etiology and prognosis of cancer pathology (Cvetkovic et al., 2009; Lo et al., 2007). Metabolomic fingerprints of lipids represents a signature yet established in cancer biology (DeBerardinis et al., 2008; Santos and Schulze 2012). Modified proteins by lipids and bioactive lipids assist network signaling of many cancer pathologies (Wymann and Schneiter, 2008)

Lipidomic analysis defines the phenotype of cells or tissues as a response to the environment or genetic changes, the level of lipids being fundamental for a defined genetic function (Van Meer, 2005; Villas-Boas and Gombert, 2006). Lipidomic biomarkers can also identify compounds of cytotoxic, aterogenic, mutagenic and carcinogenic origin (Seppänen-Laakso and Oresic, 2009).

Analytical platforms based on Chromatography coupled with Mass Spectrometry (MS) are frequently used in Lipidomics to provide the sensitive and reproducible detection of hundreds metabolites in a single biofluid or tissue sample. Biofluids, such as blood serum and plasma are the most frequent materials utilized for clinical diagnosis, to measure the level of lipids used for prediction of cancer progression (Jelonek et al., 2013; Ghahremanfard et al., 2015; Chawda et al., 2011).

Many protocols for serum- and plasma-based metabolic profiling apply gas chromatographyMS (GC-MS) and high performance liquid chromatography (HPLC) coupled with MS. Such protocols include biofluid collection, sample preparation, data acquisition, data pre-processing and quality assurance (Dunn et al., 2011)

The most suitable tool to investigate small lipid metabolites as potential biomarkers is HPLC-MS due to its high sensitivity, specificity, ease handling of samples and large number of metabolites detected (Gika et al., 2014). Two strategies can be applied: untargeted metabolomics which measures all the metabolites in a sample, screening a specific sample fingerprint and/or the targeted metabolomics that measure a limited number of metabolites and quantify their concentrations (Han and Gross, 2003; Dunn et al., 2011).

The aim of this study is to determine a lipidomic profile of lipids from the blood serum of patients with benign hyperplasia and prostate cancer, based on liquid chromatography coupled with mass spectrometry (HPLC-MS) and unsupervised biostatistics to identify the discriminations between the pathological and healthy state of lipidomic phenotypes.

\section{MATERIALS AND METHODS}

Human blood samples were collected according to a protocol approved by the Bioethical Commission Board from the University of Medicine and Pharmacy "Iuliu Hatieganu" Cluj-Napoca and after the patients' written consent prior to be included in this study. All blood samples were collected from patients registered at the Municipal Clinical Hospital Cluj-Napoca, Romania (in 2015) previously diagnosed and histologically classified with benign prostatic hyperplasia (BPH) $(n=40$, mean age $63.92 \pm 7.8$ years old), prostate sample taken before biopsy (PCa-b) $(\mathrm{n}=51$, mean age $65.67 \pm 7.5$ years old), prostate sample taken after surgery (PCa-s) $(n=38$, mean age $66.05 \pm 5.5$ years old), comparatively with control subjects ( $\mathrm{n}=12$, 
mean age $50 \pm 6.4$ years old). The pathologic groups BPH, PCa-b, PCa-s were characterized previously by different clinical parameters, including PSA values and Gleason scores (data not shown). The blood serum was obtained by standard procedure after coagulation for 30 minutes at room temperature and centrifugation, $10 \mathrm{~min}$ at $2000 \mathrm{~g}$.

Lipids were extracted according to Bligh and Dyer method (Bligh and Dyer, 1959). A volume of $0.1 \mathrm{ml}$ serum was mixed with $0.2 \mathrm{ml}$ methanol, then vortexed for $20 \mathrm{~s}$, and $1.66 \mathrm{ml}$ chloroform was added and vortexed for 20 s. Finally, a volume of $0.1 \mathrm{ml}$ water was added to induce the phase separation and centrifugated at $8000 \mathrm{~g}$ for 10 min. The lipid-chloroform phase was collected and evaporated, then reconstituted in $500 \mu \mathrm{l}$ of chromatographic eluent ACN/IPA/H2O (65:30:5, $\mathrm{v} / \mathrm{v}$ ) and ultrasonicated for $10 \mathrm{~min}$. After filtration through $0.2 \mu \mathrm{m}$ PTFE filters, the LC-MS analysis was performed.

Aliquots of $5 \mu \mathrm{l}$ of each extract were subjected to HPLC-MS on a Bruker DaltonicsMaXis Impact equipment with a Thermo Scientific HPLC UltiMate 3000 system including a quaternary pump delivery system DionexUltiMate and MS detection. The separation was done on a C18 reverse-phase column [ $5 \mu \mathrm{m}, 2.1 \times 100 \mathrm{~mm}]$, (Acclaim, Dionex) maintained at $55^{\circ} \mathrm{C}$.

The mobile phases were: A: water: acetonitrile (60:40) containing $0.1 \%$ formic acid and $10 \mathrm{mM}$ ammonium formate and $\mathrm{B}$ : isopropyl alcohol: acetonitrile (90:10) containing $0.1 \%$ formic acid and $10 \mathrm{mM}$ ammonium formate. The gradient used: $75 \%$ A: $25 \%$ B followed by linear gradient to $50 \% \mathrm{~A}: 50 \% \mathrm{~B}$ at $4 \mathrm{~min}$, then a gradient from $50 \% \mathrm{~A}$ to $3 \% \mathrm{~A}: 97 \% \mathrm{~B}$ at $19 \mathrm{~min}$, isocratic on $3 \%$ A: $97 \%$ B for 4 min and then returned to the initial condition $75 \%$ A: $25 \%$ B at 24 min for $4 \mathrm{~min}$. The flow rate was $0.260 \mathrm{ml} / \mathrm{min}$.

Mass spectrometry was performed on a Bruker Daltonics MaXis Impact Q-TOF operating in positive ion mode $\left(\mathrm{ESI}^{+}\right)$. The mass range was set between $50-1000 \mathrm{~m} / \mathrm{z}$. The nebulizing gas pressure was set at 2.8 bar, the drying gas flow at $12 \mathrm{~L} / \mathrm{min}$, the drying gas temperature at 300 ${ }^{\circ} \mathrm{C}$. Before each chromatographic run, a calibrant solution of sodium formate was injected.

TofControl 3.2 and Data Analysis 4.2 (Bruker Daltonics) software were used for the control of the instrument and data processing.

The algorithm Find Molecular Features (FMF) was applied on data in Data Analysis software and then Profile Analysis 2.0 (Bruker) for data pre-processing, alignment, bucketing (retention time range from 1' to 28' was used for bucket generation) and normalization (Sum of bucket values in analysis algorithm) was done to obtained proper matrix for biostatistics and bioinformatics analysis. Advance bucketing was done using the parameters obtained automatically from the time alignment. Only metabolites detected in more than $80 \%$ of the samples were selected to obtain the

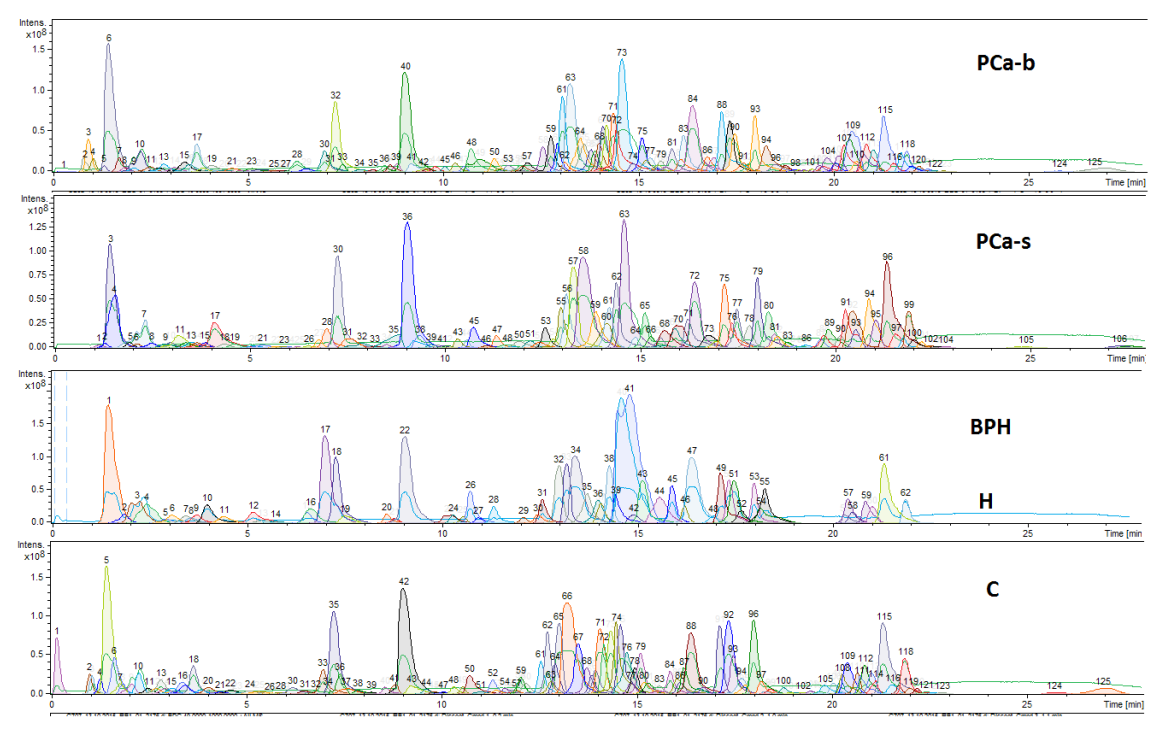

Fig.1. Comparative image of Dissected chromatograms of serum samples from C ( control), PCa-b (Biopsy cancer), PCa-s ( Surgery cancer) and BPH 
matrix utilized for the unsupervised biostatistics and multivariate analysis, by Principal Component Analysis (PCA) and Cluster Analysis (CA). The Unscrambler X 10.1 (CAMO Software AS, Norway) software was also used, importing the matrix data and applying the multivariate analysis like PCA and CA. The distance method used for CA was Euclidean and the linkage method was Average.

\section{RESULTS AND DISCUSSION}

\section{Chromatographic profile of serum lipids}

Fig.1 shows the fingerprints of the blood serum lipid extracts, comparatively, from C ( control), PCa-b (Biopsy cancer), PCa-s ( Surgery cancer) and BPH patients.

The number of lipid molecules which were separated by HPLC ranged from $62(\mathrm{BPH})$ to 123 , separated in 23-25 min. Around six groups of molecules were separated, according to their polarity and molecular weight: very polar lipids and non-lipid molecules from min 1 to 5, polar lipids from min 6 to 9 ( group 2), semipolar lipids from min 12-15 (group 3), neutral lipids from min 12 to 16 ( group 4) and nonpolar lipids from min 20 to 23 ( group 5). The identification of these lipids was done by comparing their ESI $+m / z$ values with the values registered in Lipidomics
Gateway (http://www.lipidmaps.org/ ) and Human Metabolomic Data Base (http://www. hmdb.ca/).

Nontargeted, unsupervised analysis of data by Principal Component Analysis

Using the Uncrambled software and the optimized parameters for data processing ( see Materials and Methods), the statistics applied for data inputs was Principal Component Analysis (PCA). In Fig. 2 there are shown the PCA scores (upper graphics) and loadings (down graphics), to discriminate between biopsy cancer vs Control (a), surgery cancer vs Control (b), Hyperplasia vs Control (c).

Fig. 2a shows 2 subgroups of biopsy cancer patients, one group having a fingerprint close to controls (marked with red circles) and one subgroup significantly different. The loadings graphic shows that 3 molecules with $\mathrm{m} / \mathrm{z}$ values of $282.281,520.344$ and 524.345 may be responsible for these differences.

Fig. $2 \mathrm{~b}$ shows also 2 subgroups, but the second one less abundant, of surgery cancer patients, one group having a fingerprint close to controls (marked with red circles) and a secondary, less abundant subgroup significantly different. The loadings graphic shows that molecules with $\mathrm{m} / \mathrm{z}$

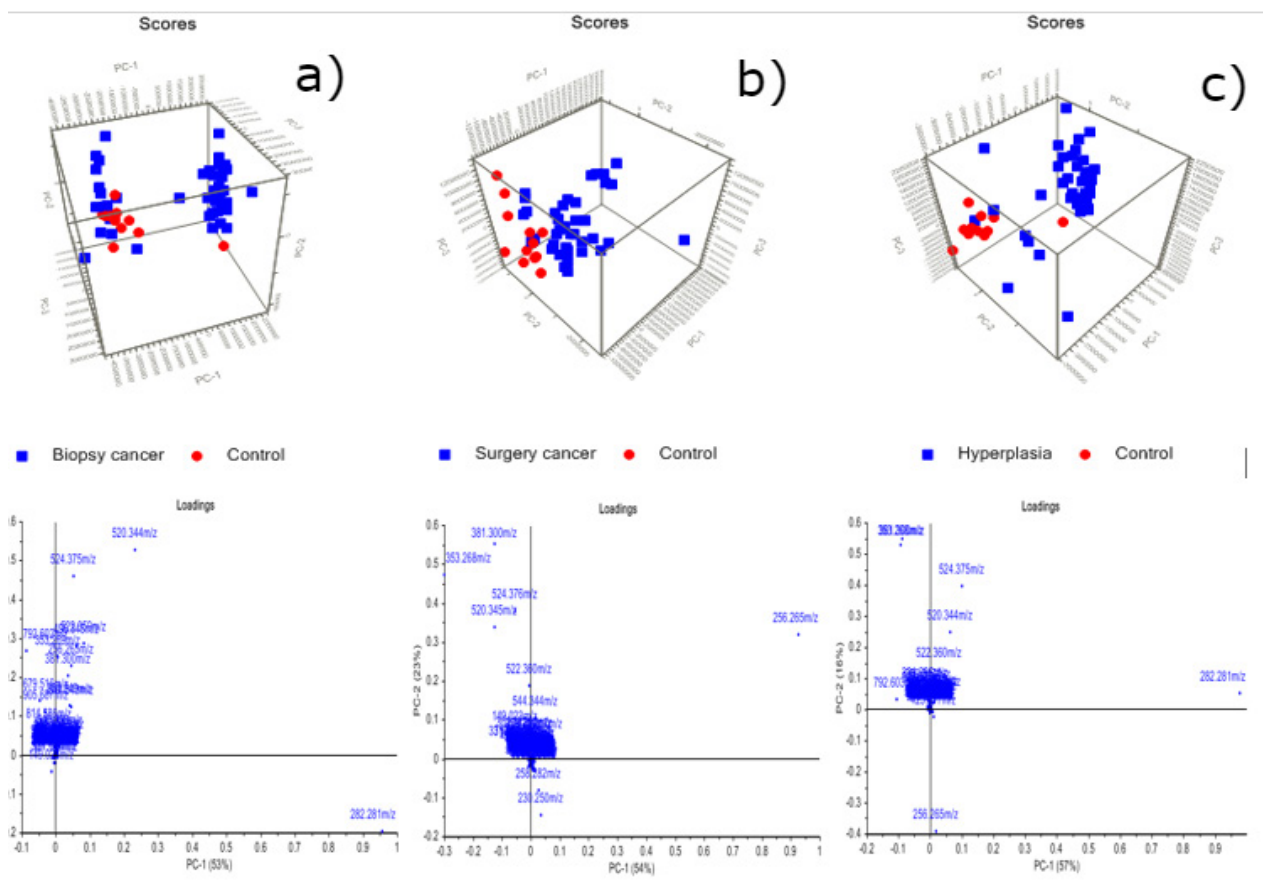

Fig. 2. PCA scores and loadings of pathological groups ( biopsy cancer, surgery cancer, hyperplasia) vs control group 
values of $256.265,381.300,353.268,520.344$, 522.360 and 524.345 are responsible for these differences.

Fig. 2c shows also 2 subgroups, a first group less abundant, having a fingerprint close to controls (marked with red circles) and a secondary, less abundant subgroup significantly different. The loadings graphic shows that molecules with $\mathrm{m} / \mathrm{z}$ values of $256.265,282.281,353.268,520.344$,
522.360 and 524.345 are responsible for these differences.

Finally, according to these data eleven molecules were selected as potential biomarkers, in all cases three similar molecules with $\mathrm{m} / \mathrm{z}$ values of $520.344,522.360$ and 524.345 (corresponding to 3 successive C18-lysophosphatidyl cholines) were responsible for the discriminations between the pathologic and control groups. The molecules with $\mathrm{m} / \mathrm{z}=282.281$ were different in biopsy and

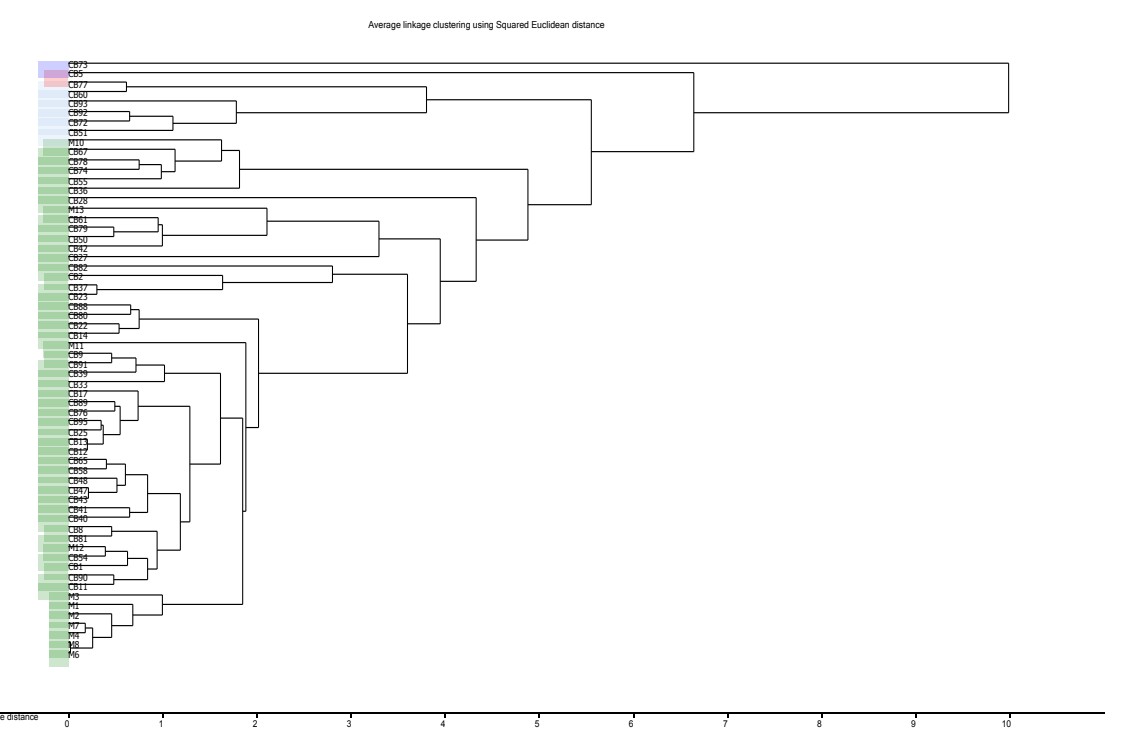

Fig. 3. Cluster dendogram based on average linkage method using Squared Euclidean distance of biopsy group (CB) and controls (M).

Fig. 4. Cluster dendogram based on average linkage method using Squared Euclidean distance of surgery group (CO) and controls (M). 
hyperplasia groups, while molecules with $\mathrm{m} / \mathrm{z}$ $=256.265,381.300,353.268$ were significantly different in surgery cancer group vs controls.

Nontargeted, supervised interpretation of data: Cluster Analysis

The Hierarchial Cluster Analysis processed the data obtained from FMF matrix considering an advanced bucketing. According to Fig.3, for biopsy cancer (marked CB) two clustering groups were obtained, in accordance with the PCA data ( Fig.2a). For controls, a good clustering was found also, excepting M10 and M11.

According to Fig.4, for surgery cancer group (marked CO) there were found more dispersed clustering groups, the $\mathrm{CO}$ samples being clustered in four subgroups.

According to Fig.5, for hyperplasia group (marked $\mathrm{H}$ ) there were found more dispersed clustering groups, spread among the controls. The significance of this clustering is that not so many statistical significant differences can be found between the hyperplasia and control groups.

\section{Identification of specific lipid biomarkers} of the pathologic groups

Using the untargeted biostatistics processed by Profile Analysis, there were identified

Fig. 5. Cluster dendogram based on average linkage method using Squared Euclidean distance of Hyperplasia group $(\mathrm{H})$ and controls $(\mathrm{M})$.

Tab. 1. Tentative assignement of main molecules corresponding specifically to pathologic groups (for abbreviations, see the text).

\begin{tabular}{ccc}
\hline $\mathrm{m} / \mathrm{z}$ & Tentative assignment & Specific to \\
\hline 316.322 & Eicosanoic acid or decanoylcarnitine & PCa-s \\
\hline 369.352 & Tetracosanoic acid & PCa-b, BPH \\
\hline 415.211 & Ascorbyl palmitate & PCa-s, PCa-b, BPH \\
\hline 432.240 & N-stearoyl phenylalanine & PCa-b \\
\hline 607.570 & DG(16:0/0:0/18:2n6) & PCa-b \\
\hline 666.627 & PS(0-16:0/12:0) & PCa-b, BPH \\
\hline 582.582 & Ceramide(d14:1/22:0(20H)) or Ceramide $(\mathrm{d} 16: 1 / 20: 0(20 H))$ & PCa-s \\
\hline 806.573 & $\mathrm{PC}(16: 0 / 22: 6)$ or $\mathrm{PC}(18: 1 / 20: 5)$ or $\mathrm{PC}(18: 2 / 20: 4)$ & PCa-s \\
\hline 801.561 & $\mathrm{TG}(16: 1 / 14: 0 / 18: 2)$ or TG(16:0/14:0/18:3) & BPH \\
\hline 760.589 & $\mathrm{PC}(16: 0 / 18: 1)$ or PC $(16: 1 / 18: 0)$ & BPH \\
\hline 810.604 & $\mathrm{PC}(18: 0 / 20: 4)$ or $\mathrm{PC}(18: 1 / 20: 3)$ or PC(18:2/20:2) & BPH \\
\hline
\end{tabular}


the specific molecules, related to a specific pathology, based on the peak areas and $\mathrm{m} / \mathrm{z}$ values (Table 1). The molecules with $\mathrm{m} / \mathrm{z}$ values of 316.3227 (corresponding to eicosanoic acid or decanoylcarnitine), 582.582 (ceramides) and 806.573 (corresponding to $\mathrm{PC}(16: 0 / 22: 6)$ or $\mathrm{PC}(18: 1 / 20: 5)$ or $\mathrm{PC}(18: 2 / 20: 4)$ are specific to surgery cancer (PCa-s). The molecules with $\mathrm{m} / \mathrm{z}$ values of 432.240 ( $\mathrm{N}$-stearoyl phenylalanine) and 607.570 DG(16:0/0:0/18:2n6) are specific to biopsy cancer (PCa-b). The molecules with $\mathrm{m} / \mathrm{z}$ values of 801.561 (TG(16:1/14:0/18:2), TG(16:0/14:0/18:3)), $760.589 \quad \mathrm{PC}(16: 0 / 18: 1)$ or $\mathrm{PC}(16: 1 / 18: 0), \quad 810.604(\mathrm{PC}(18: 0 / 20: 4)$ or $\mathrm{PC}(18: 1 / 20: 3)$ or $\mathrm{PC}(18: 2 / 20: 2)$ are specific to hyperplasia (BPH). The molecules 369.352 (Tetracosanoic acid) and 666.627 (PS(O16:0/12:0) are specific to groups of biopsy cancer (PCa-b) and hyperplasia (BPH). The molecule with $\mathrm{m} / \mathrm{z}=415.211$ (Ascorbyl palmitate) is common to all groups.

\section{CONCLUSION}

By an advanced technique HPLC coupled with Mass Spectrometry and an optimized data processing and analysis we were able to separate and identify hundreds if lipid molecules in blood serum, as candidates for prostate pathologies (hyperplasia and cancer).

The statistical unsupervised analysis based on PCA scores and loadings, using the Uncrambled softaware, showed a good discrimination between the two cancer groups of patients (after biopsy and after surgery) and for benign hyperplasia patients against controls, based on the comparison of peak areas. The molecules responsible for such discriminations were identified to be mainly represented by lysophospatidylcholines. By Cluster Analysis, the dendograms showed good statistical clustering of samples, especially for cancer patients against controls and less clustered for hyperplasia.

Using a Profile Analysis software, the identification of lipid biomarkers for specific pathologic groups was possible. Molecules belonging to phospholipid family and diacyl / triacylglycerides or ceramides or carnitines can be considered potential biomarkers for hyperplasia and prostate cancer.
Acknowledgments. The experimental part of this work was supported by the Center for Applied Biotechnology CCD-BIODIATECH Cluj-Napoca, Romania.

\section{REFERENCES}

1. Bligh EG and Dyer W J (1959). A rapid method of total lipid extraction and purifi cation. Can J Biochem Physiol 37: 911 - 917.

2. Chawda JG, Jain SS, Patel HR, Chaduvula N, Patel K (2011). The relationship between serum lipid levels and the risk of oral cancer. Indian Journal of Medical and Paediatric Oncology 32(1): 34-37.

3. Cvetkovic Z, Cvetkovic B, Petrovic M, Ranic M, DebeljakMartarcic J, Vucic V et al. (2009). Lipid profile as a prognostic factor in cancer patients. J BUON 14 (3):501506.

4. Deberardinis RJ, Lum JJ, Hatzivassiliou G, Thompson CB (2008) The biology of cancer: Metabolic reprogramming fuels cell growth and proliferation. Cell Metab 7: 11-20.

5. Dunn WB, Broadhurst D, Atherton HJ, Goodacre R, GriYn JL (2011) Systems level studies of Mammalian metabolomes: the roles of mass spectrometry and nuclear magnetic resonance spectroscopy. Chemical Society Reviews 40(1): 387-426

6. Feng L, Prestwich GD (2005). Functional lipidomics. Dekker-CRC, New York,p. 1-329

7. Ferlay J, Soerjomataram I, Ervik M, Dikshit R, Eser Sc, Mathers C, Rebelo M, Parkin Dm, Forman D, Bray F (2013). GLOBOCAN, 2012 v1.0, Cancer Incidence and Mortality Worldwide: IARC CancerBase No. 11 International Agency for Research on Cancer, Lyon, France, p. 2015

8. Ghahremanfard F, Mirmohammadkhani M, Shahnazari B, Gholami G \& Mehdizadeh J (2015). The valuable role of measuring serum lipid profile in cancer progression. Oman Medical Journal, 30(5): 353-357.

9. Gika HG, Theodoridis G, Plumb AR, Wilson SID (2014) Current practice of liquid chromatography-mass spectrometry in metabolomics and metabonomics. Journal of Pharmaceutical Biomedical Analysis 87: 12-25

10. Han X (2009). Lipidomics: developments and applications. J. Chromatogr. B 877: 2663-2669.

11. Han X, Gross RW (2003). Global analyses of cellular lipidomes directly from crude extracts of biological samples by ESI mass spectrometry: a bridge to lipidomics. J Lipid Res 44:1071-1079.

12. Jelonek K, Ros M, Pietrowska M, \& Widlak P (2013). Cancer biomarkers and mass spectrometry-based analyses of phospholipids in body fluids. Clinical Lipidology, 8(1): 137-150.

13. Lo AC, Soliman AS, El-Ghawalby N, Abdel-Wahab M, Fathy O, Khaled HM (2007). Lifestyle, occupational, and reproductive factors in relation to pancreatic cancer risk. Pancreas 35(2):120-129.

14. Lokhov PG, Dashtiev MI, Moshkovskii SA, Archakov AI (2010) Metabolite profiling of blood plasma of patients with prostate cancer. Metabolomics 6: 156-163 
15. Mishra A, Verma M (2010). Cancer biomarkers: Are we ready for the prime time? Cancers, 2(1), 190-208.

16. Osl M, Dreiseitl S, Pfeifer B, Weinberger K, Klocker $H$, Bartsch G, Schäfer G, Tilg B, Graber A, Baumagartner C (2008). A new rule-based algorithm for identifying metabolic markers in prostate cancer using tandem mass spectrometry. Bioinformatics Original Paper 24(24): 2908-2914.

17. Punglia RS (2003). Effect of verification bias on screening for prostate cancer by measurement of prostate-specific antigen. N Engl J Med 349: 335-342.

18. Rolim AEH (2015). Lipidomics în the study of lipid metabolism: Current perspectives în the omic sciences. Gene 554, 131-139.

19. Santos CR \& Schulze A (2012). Lipid metabolism in cancer FEBS Journal 279(15): 2610-2623.

20. Seppänen-Laakso T, Oresic M (2009). How to study lipidomes. J Mol Endocrinol. 42: 185-190.

21. Teahan O, Bevan CL, Waxman J, Keun HC (2011) Metabolic signatures of malignant progression in prostate epithelial cells. The International Journal of Biochemistry \&Cell Biology 43: 1002-1009.
22. Van Meer G (2005). Cellular lipidomics. EMBO J 24: 3159 3165.

23. Verma M, Banerjee HN (2015). Metabolomic approaches in cancer epidemiology. Disease 3: 167-175.

24. Vicini FA, Vargas C, Abner A, Kestin L, Horwitz E, Martinez A (2005). Limitations in the use of serum prostate specific antigen levels to monitor patients after treatment for prostate cancer. The Journal of Urology 173(5): 14561462.

25. Villas-Boas SG, Gombert AK (2006). Análise do Metaboloma: Una ferramenta biotecnológica emergente na era pósgenômica. Biotecnol Cienc Desenvolv 36: 5869.

26. Wenk MR (2005). The emerging field of lipidomics. Nat Rev Drug Discov 4, 594-610.

27. Wolf C, Quinn PJ (2008). Lipidomics: practical aspects and applications. Prog Lipid Res 47:15-36.

28. Wymann MP, Schneiter R (2008). Lipid signalling in disease, Nat Rev Mol Cell Biol 9:162-176.

29. Zhang A, Yan G, Han Y, Wang X (2014). Metabolomics approaches and applications in prostate cancer research. Appl Biochem and Biotechnol 174(1): 6-12. 